\title{
PH-7
}

\section{Understanding of FWM Behavior in 2-D Time-Spreading Wavelength- Hopping OCDMA Systems}

\author{
Taher M. Bazan \\ Egyptian Armed Forces
}

\begin{abstract}
The behavior of four-wave mixing (FWM) in 2-D time-spreading wavelength-hopping (TW) optical code-division multiple-access (OCDMA) systems with coherent light sources is introduced. The analysis is performed in terms of several key system parameters, in particular, transmitted power per chip, wavelength spacing, number of active users, code weight,fiber type, and transmission length. To accomplish such study, the number of generated FWM components should be calculated first. Thus, for the first time, a general form for the FWM power generated in 2-D TW OCDMA systems is presented and explicit formulas for the number of FWM products that fall on each signal chip for symmetric codes are given.
\end{abstract}

Keywords: Optical code division multiplexing, 2-D Wavelength-time coding, Four-wave mixing

\section{Introduction}

Over the past two decades, 2-D TW OCDMA techniques in optical fiber networks have received considerable research interest because of their ability to increase the system user capacity, provide multiple-user access, employ asynchronous operation and support high speed applications [1-2]. In 2-D TW OCDMA schemes, each user is assigned a signature code which is spread in time and wavelength in order to minimize the multiple access interference and to maximize the number of subscribers. Most research in such area are directed to investigate the system performance under different code families, transmitter and receiver architectures while neglecting the effect of optical fiber nonlinearity.

FWM process is an example of third-order nonlinear effects arising from intensitydependent variations in the refractive index in a silica fiber [3]. FWM has been extensively studied in wavelength division multiplexing (WDM) systems in which it results in FWMinduced crosstalk and leads to system degradation especially in high power region [4-5]. If the new FWM frequencies coincide with the existing ones, this may lead to a coherent inband crosstalk while other FWM components that fall in between the desired frequencies can lead to incoherent out-of-band crosstalk. In fact, the effect of coherent crosstalk degrades system performance much more severely. However, there have been limited reports on the influence of FWM in OCDMA systems and its impact was not yet investigated in details [6-7]. This paper will give more understanding on the behavior of FWM in 2-D TW OCDMA systems. The paper is structured as follows: Section 2 
introduces the definition and a general expression for estimation the FWM power arise in 2-D OCDMA systems. Empirical formulas, for the first time, for the number of FWM products that fall on the desired user chips are presented in Section 3. In Section 4 numerical results are discussed and finally the paper is concluded in Section 5.

\section{FWM in TW OCDMA}

Through an FWM process, electromagnetic fields of three different frequencies $f_{r}, f_{p}$, and $f_{q}$ of power $P$ propagate simultaneously through optical fiber length $L$ to generate new frequency $f_{r p q}=f_{r}+f_{p}-f_{q}$. A well-known formula is utilized to determine the FWM power as follow [3]:

$$
P_{\text {FWM }}=\frac{\eta}{9} d^{2} \gamma^{2} P_{r} P_{p} P_{q} L_{\text {eff }}^{2} \exp (-\alpha L)
$$

where $\gamma$ is the third-order non-linear susceptibility of the optical fiber, $L_{\text {eff }}$ is effective fiber length, $d$ is the degeneracy factor ( $d=3$ or 6 for $r=p$ or $r \neq p$, respectively), $\alpha$ is the fiber loss coefficient, and $\eta$ is FWM efficiency expressed by [3]

$$
\eta=\frac{\alpha^{2}}{\Delta \beta+\alpha^{2}}\left\{1+\frac{4 e^{-\alpha L} \sin ^{2}\left(\frac{\Delta \beta . L}{2}\right)}{\left(1-e^{-\alpha L}\right)^{2}}\right\}
$$

where $\Delta \beta$ is the phase mismatch and is given in terms of frequency difference by

$$
\Delta \beta=\frac{2 \pi \lambda_{q}^{2}}{c} \cdot\left|f_{r}-f_{q}\right| \cdot\left|f_{p}-f_{q}\right| \cdot\left[D_{c}+\frac{\lambda_{k}^{2}}{2 c}\left(\left|f_{r}-f_{q}\right|+\left|f_{p}-f_{q}\right|\right) \frac{d D_{c}\left(\lambda_{q}\right)}{d \lambda}\right]
$$

Here, $D_{\mathrm{c}}$ is the chromatic dispersion whereas $d D_{\mathrm{c}} / d \lambda$ is the dispersion slope. Note that the non-linear coefficient $\gamma$ can be defined as follow:

$$
\gamma=\frac{2 \pi n_{2}}{\lambda \cdot A_{\text {eff }}}
$$

where $\lambda$ is the wavelength and $A_{\text {eff }}$ is the core effective area of fiber. $n_{2}$ is the fiber nonlinear refractive index related to nonlinear susceptibility $\chi_{1111}$, refractive index of fiber core $n_{2}$ and vacuum speed of light $c$ by the relation:

$$
n_{2}=\frac{48 \pi^{2}}{c n^{2}} \chi_{1111}
$$

In WDM systems, each user is assigned a unique wavelength, so the number of FWM products depends mainly on number of users (or wavelengths) but 2-D TW OCDMA systems have the property that each user data is encoded with a signature code which has a number of chips with different wavelengths according to a particular pattern. Consequently, the number of generated FWM products in 2-D TW OCDMA systems depends not only on the number of users but also on the number and pattern of the utilized wavelengths. This work will focus on the most important situation when some of the generated FWM components coincide with any wavelength of the selected user chips that yields to interference. Therefore, the general form for the generated FWM power in 2-D TW OCDMA due to $K$ active users and $w$ available wavelengths with symmetric codes after distance $L$ can be defined as 


$$
P_{F W M}^{O C D M A}=\sum_{\substack{i \geq 2 \\ A, B, C \in i}}^{K} \sum_{s=1}^{w} h P_{F W M}(s, i)=\sum_{\substack{i \notin 2 \\ A, B, C \in i}}^{K} \sum_{\delta=1}^{w} h \frac{\eta}{9} d^{2} \gamma^{2} P_{r}^{A} P_{p}^{B} P_{q}^{C} L_{\text {eff }}{ }^{2} \exp (-\alpha L)
$$

where $h$ is the probability of existence of a given combination $f_{r}, f_{p}$, and $f_{q}$ at one time slot of the code length. Assuming and probability of sending bit " 1 " is $1 / 2$, then, $h=1 / 8 N^{2}$ or $1 / 4 N$ for the nondegenerate and degenerate cases respectively, where $N$ is the code weight. In fact, two cases are associated with FWM condition; completely nondegenerate and partially degenerate events. In the former case, three frequencies originating from three users are required to produce FWM while in the latter case, mixing of two waves from different users is sufficient for FWM production. According to Eq. (6), the strength of each FWM product is weighted according to its mixing efficiency and probability of existence. The summations in Equation (6) will be calculated as shown in the next section.

\section{Number of FWM Products in TW OCDMA}

In this section, explicit formulas for the total number of FWM products employing TW OCDMA codes with equally spaced frequencies (or consequently wavelengths) satisfying $f_{s}=f_{r}+f_{p}-f_{q}$ are presented. The analysis here is devoted to codes in which all the available chip frequencies $\left(f_{1}, f_{2}, \ldots, f_{w}\right)$ are utilized in each code weight $w$ ( $w$ is a prime number); such as prime hop codes [1]. It is worth mentioned that time spreading mechanism is not a factor in estimating the number of generated FWM products. Nevertheless, in a way similar to [4], let $k$ is the number of active users and consider only the FWM components that fall on the operating wavelengths, then, the frequency combinations can be classified into five main groups as follows:

\section{a. Group I}

Consider the subscript denotes a particular chip frequency whilst the superscript represents the user number from which user this frequency originate. In this group, mixing frequencies from any three users except the selected or desired user are satisfying FWM condition, i.e. such a group represents a completely nondegenerate case such that

$$
f_{r}^{A}+f_{y}^{B}-f_{q}^{C}=f_{s}^{i n t}
$$

$\forall \mathrm{A}, \mathrm{B}, \mathrm{C}=1,2, \ldots, K-1$, where $K$ is the number of users and $\mathrm{A} \neq \mathrm{B} \neq \mathrm{C}$, whereas $r, p, q$, and $s$ are the operating chip frequencies (or wavelengths) in the code family, i.e., $r, p, q$, and $s$ $=1,2, \ldots, w . f_{s}^{\text {int }}$ is the new generated FWM frequency and is matched to one of the hopped frequencies allocated to the desired user. Also, the case of $f_{1}^{A}+f_{1}^{B}-f_{1}^{C}$, for example, is not considered as Self-phase modulation since the mixing of the same frequencies occurs from different users. However, the number of FWM products at any frequency chip index $n(1,2, \ldots w)$ of the desired user for this case based on tetrahedral numbers can be given by:

$$
N_{F W M}^{I}(n, k)=\left\{\begin{array}{cc}
\frac{(K-1)(K-2)(K-3)}{3}\left[\frac{3\left(3 w^{2}+1\right)}{4}-3(M-n)^{2}\right] & n=1,2, \ldots, w-\{M\} \\
\frac{\left(3 w^{2}+1\right)(K-1)(K-2)(K-3)}{4} & n=M
\end{array}\right.
$$

where $M$ is the index of the middle chip wavelength; $M=(w+1) / 2$. 


\section{b. Group II}

Here, in such a group, the mixing frequencies of any two users with the desired user will generate FWM products such that

$$
f_{r}^{A}+f_{p}^{B}-f_{q}^{i n t}=f_{s}^{i n t}
$$

$\forall \mathrm{A}, \mathrm{B}=1,2, \ldots, K-1$, and $\mathrm{A} \neq \mathrm{B}$, again $r, p, q$, and $s$ are the available chip frequencies in the code family, i.e., $r, p, q$, and $s=1,2, \ldots, w$. The FWM products for case II can be estimated as follow

$$
N_{F W M}^{I I}(s, k)=\left\{\begin{array}{cc}
(k-1)(k-2)\left[\frac{3 w^{2}+1}{4}-(M-n)^{2}\right] & s=1,2, \ldots, w-\{M\} \\
\frac{\left(3 w^{2}+1\right)(k-1)(k-2)}{4} & s=M
\end{array}\right.
$$

\section{c. Group III}

The FWM products in this category are due to the mixing between identical chip frequency from the same user and another one from a different user (partially degenerate case); i.e.

$$
f_{r}^{A}+f_{r}^{A}-f_{q}^{B}=f_{s}^{i n t}
$$

In this group, it is obvious that any two users other than the desired one may be sufficient for FWM generation and the number of FWM components can be expressed as

$$
N_{F W M}^{I I I}(s, k \geq 3)= \begin{cases}\frac{w+1}{2} \times(k-1) \times(k-2) & s=\text { odd } \\ \frac{w-1}{2} \times(k-1) \times(k-2) & s=\text { even }\end{cases}
$$

\section{d. Group IV}

Group IV is similar to group III except that two mixed waves are sufficient to generate non-degenerate FWM so that

$$
f_{r}^{A}+f_{r}^{A}-f_{q}^{i n t}=f_{s}^{i n t}
$$

The following formula estimating the number of FWM components for two users can be applied

$$
N_{F W M}^{I V}(s, k)= \begin{cases}\frac{(w+1) \times(k-1)}{2} & s=\text { odd } \\ \frac{(w-1) \times(k-1)}{2} & s=\text { even }\end{cases}
$$

\section{e. Group V}

The last group is devoted to FWM products resulted from three users including the desired user as follow

$$
f_{r}^{i n t}+f_{p}^{A}-f_{q}^{B}=f_{s}^{i n t}
$$

The number of FWM products for a given wavelength index $n$ when $k=3$ is 
Military Technical College

Kobry Elkobbah,

Cairo, Egypt

April 19-21,2016 $8^{\text {th }}$ International Conference on

Mathematics and Engineering Physics (ICMEP-8)

$$
N_{F W M}^{V}(s, k=3)=\left\{\begin{array}{cc}
2\left[P^{2}+4 P-3+\sum_{b=0}^{M-2} 4(b-1)\right] & s=M \\
N_{F W M}^{V}(M, k=3)-2\left\{6+6(M-n-1)+\sum_{b=0}^{M-n-1} 4(b-1)\right\} & s=\text { else }
\end{array}\right.
$$

where $N_{F W M}^{V}(M, k=3)$ is the number of FWM components at the middle chip frequency of the existing frequencies when $K=3$, i.e. at $(w+1) / 2$. Similarly to case III, based on triangular numbers, the number of FWM is

$$
N_{F W M}^{V}(s, k>3)=N_{F W M}^{V}(s, k=3) \times \frac{(k-1) \times(k-2)}{2}
$$

Finally, a closed form of the total number of FWM components can be calculated by the sum of Equations (8, 10, 12, 14 and 17), whereas any other possible combinations are related to Self-Phase modulation and Cross-Phase modulation.

As an illustrative example, suppose we have $K=4$ users employing prime hop codes with code weight of $w=3$, then, index of the middle wavelength $M=2$. Consider the chip wavelengths $\lambda_{1}, \lambda_{2}$, and $\lambda_{3}$ have equal wavelength space $\Delta \lambda$ such that their corresponding frequencies are $f_{1}=f-\Delta f, f_{2}=f$, and $f_{3}=f+\Delta f$ respectively. Thus, the user $\# 1$ has code weight of $f_{1}^{1}, f_{2}^{1}, f_{3}^{1}$. Similarly, chip indices of user \#2, can be represented by $f_{1}^{2}, f_{2}^{2}, f_{3}^{2}$ and so on. Thus, one possible mixing combinations to generate new FWM with frequency identical to the first chip frequency of the desired code can be expressed as $f_{1}^{1}=\left(f_{1}^{2}, f_{1}^{3}, f_{1}^{4}\right)$. All possible mixing combination to generate new FWM at $f_{1}^{1}$ in Group I is presented in Table 1.

Table 1. All possible mixing combination to generate new FWM at $f_{1}^{1}$ in Group I

\begin{tabular}{|c|c|c|c|c|c|}
\hline$\left(f_{1}^{2}, f_{1}^{3}, f_{1}^{4}\right)$ & $\left(f_{2}^{2}, f_{1}^{3}, f_{2}^{4}\right)$ & $\left(f_{3}^{2}, f_{1}^{3}, f_{3}^{4}\right)$ & $\left(f_{1}^{2}, f_{3}^{3}, f_{3}^{4}\right)$ & $\left(f_{1}^{3}, f_{1}^{2}, f_{1}^{4}\right)$ & $\left(f_{2}^{3}, f_{1}^{2}, f_{2}^{4}\right)$ \\
\hline$\left(f_{1}^{3}, f_{1}^{4}, f_{1}^{2}\right)$ & $\left(f_{2}^{3}, f_{1}^{4}, f_{2}^{2}\right)$ & $\left(f_{3}^{3}, f_{1}^{4}, f_{3}^{2}\right)$ & $\left(f_{1}^{3}, f_{3}^{4}, f_{3}^{2}\right)$ & $\left(f_{1}^{4}, f_{1}^{3}, f_{1}^{2}\right)$ & $\left(f_{2}^{4}, f_{1}^{3}, f_{2}^{2}\right)$ \\
\hline$\left(f_{1}^{4}, f_{1}^{2}, f_{1}^{3}\right)$ & $\left(f_{2}^{4}, f_{1}^{2}, f_{2}^{3}\right)$ & $\left(f_{3}^{4}, f_{1}^{2}, f_{3}^{3}\right)$ & $\left(f_{1}^{4}, f_{3}^{2}, f_{3}^{3}\right)$ & $\left(f_{1}^{2}, f_{1}^{4}, f_{1}^{3}\right)$ & $\left(f_{2}^{2}, f_{1}^{4}, f_{2}^{3}\right)$ \\
\hline$\left(f_{1}^{2}, f_{2}^{3}, f_{2}^{4}\right)$ & $\left(f_{2}^{2}, f_{2}^{3}, f_{3}^{4}\right)$ & $\left(f_{3}^{3}, f_{1}^{2}, f_{3}^{4}\right)$ & $\left(f_{1}^{3}, f_{3}^{2}, f_{3}^{4}\right)$ & $\left(f_{1}^{3}, f_{2}^{2}, f_{2}^{4}\right)$ & $\left(f_{2}^{3}, f_{2}^{2}, f_{3}^{4}\right)$ \\
\hline$\left(f_{1}^{3}, f_{2}^{4}, f_{2}^{2}\right)$ & $\left(f_{2}^{3}, f_{2}^{4}, f_{3}^{2}\right)$ & $\left(f_{3}^{4}, f_{1}^{3}, f_{3}^{2}\right)$ & $\left(f_{1}^{4}, f_{3}^{3}, f_{3}^{2}\right)$ & $\left(f_{1}^{4}, f_{2}^{3}, f_{2}^{2}\right)$ & $\left(f_{2}^{4}, f_{2}^{3}, f_{3}^{2}\right)$ \\
\hline$\left(f_{1}^{4}, f_{2}^{2}, f_{2}^{3}\right)$ & $\left(f_{2}^{4}, f_{2}^{2}, f_{3}^{3}\right)$ & $\left(f_{3}^{2}, f_{1}^{4}, f_{3}^{3}\right)$ & $\left(f_{1}^{2}, f_{3}^{4}, f_{3}^{3}\right)$ & $\left(f_{1}^{2}, f_{2}^{4}, f_{2}^{3}\right)$ & $\left(f_{2}^{2}, f_{2}^{4}, f_{3}^{3}\right)$ \\
\hline
\end{tabular}

\section{Results and discussion}

In this section, numerical results are presented to quantify the number of FWM sidebands produced in 2-D WT OCDMA schemes and highlight the sensitivity of FWM noise power to system parameters according to the above mentioned discussion. The total number of FWM products as a function of number of users for different prime hop code weights with equally spaced wavelengths is depicted in Fig. 1. 


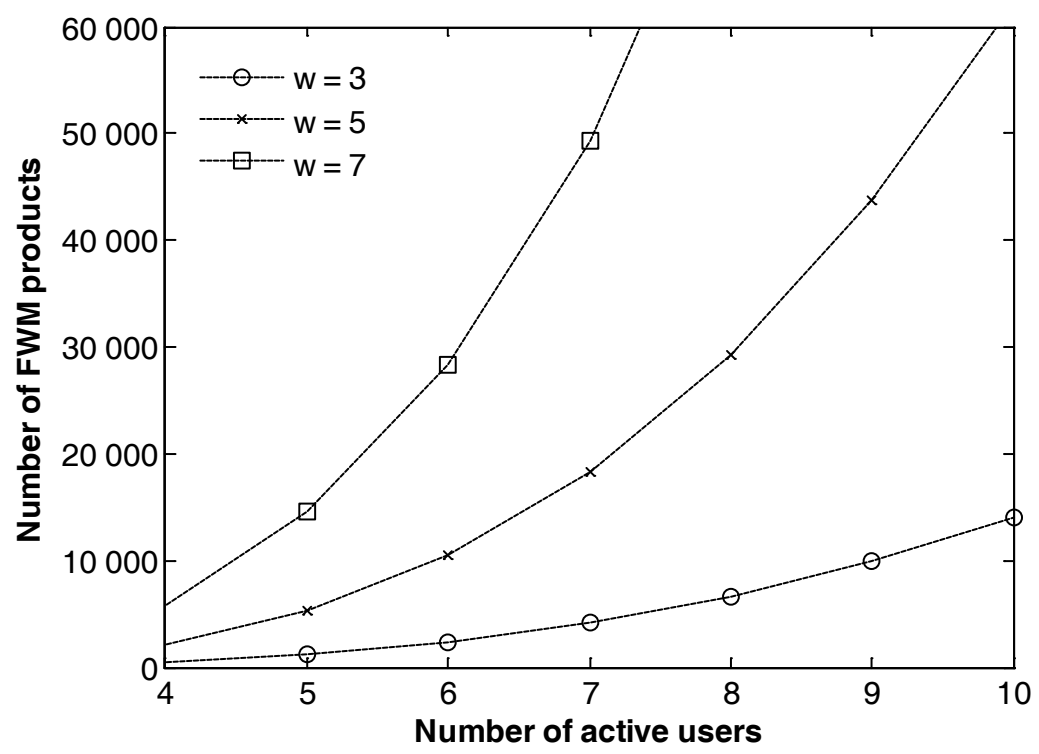

Fig. 1 Number of FWM products versus the number of users for different code weights

For fixed code weight, large number of sidebands is generated (falling on the desired chips) even for low number of users. For instance, at $K=5$ and $w=5$, the number of FWM components is 5308 terms and when the number of users is doubled, it gives 11 times more FWM products. Furthermore, for the same number of users, small increment in the code weight results in exponential increase in the interfering products; more than 4 times rise in the FWM products when the weight increases from 3 to 5 at $K=6$. However, Fig. 2 gives more scope on the number of FWM products that fall on each desired chip. The middle wavelength, in general, has higher number of generated products than other utilized wavelengths in the code and lower number of products arises when go away from the middle wavelength as shown in Fig. 2.In Fig. 3, the total FWM-noise power for various injected power values into the fiber is plotted as a function of number of active users. The figure shows that as the number of users increase, the FWM-noise power also increases. In addition, increasing the values of injected users' powers imposes more FWM-noise power but further increase in the launched power through the fiber will not lead to a high noise power change. For example, when $K=14$, the increasing injected power from 1 to $5 \mathrm{~mW}$ leads to an increase of FWM-noise power by $20 \mathrm{~dB}$ but increasing extra power to from 5 to $10 \mathrm{~mW}$ induces noise-power difference in the level of $10 \mathrm{~dB}$. 


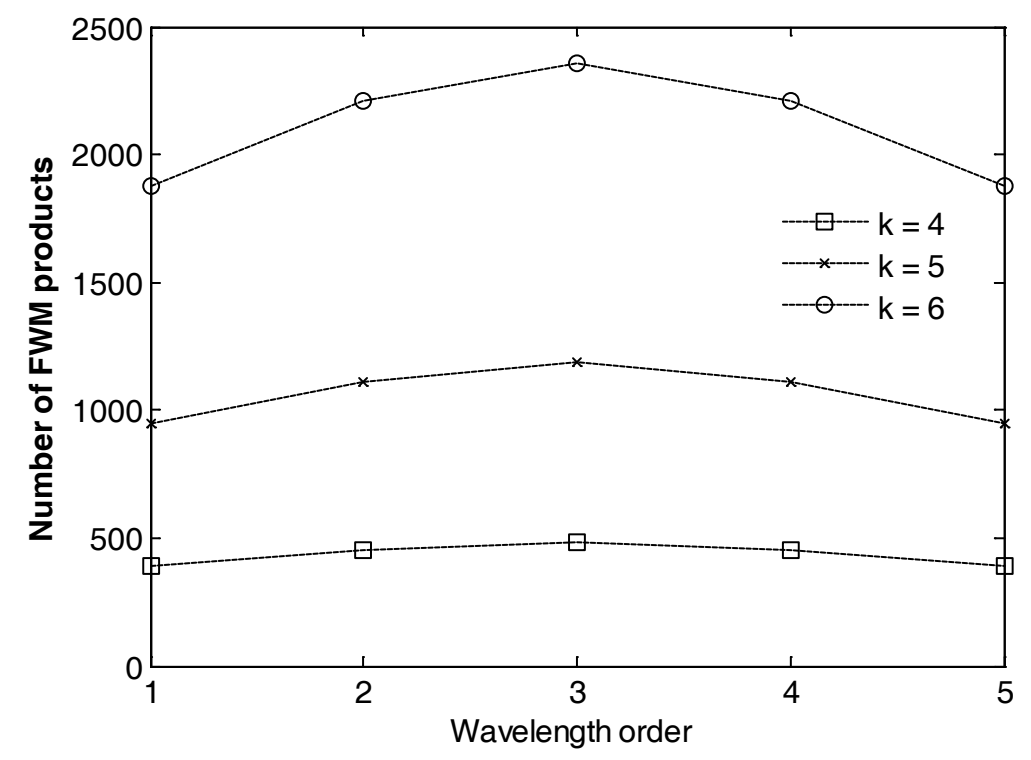

Fig. 2 Number of FWM products versus each wavelength in the code weight $(w=5)$ for different number of users

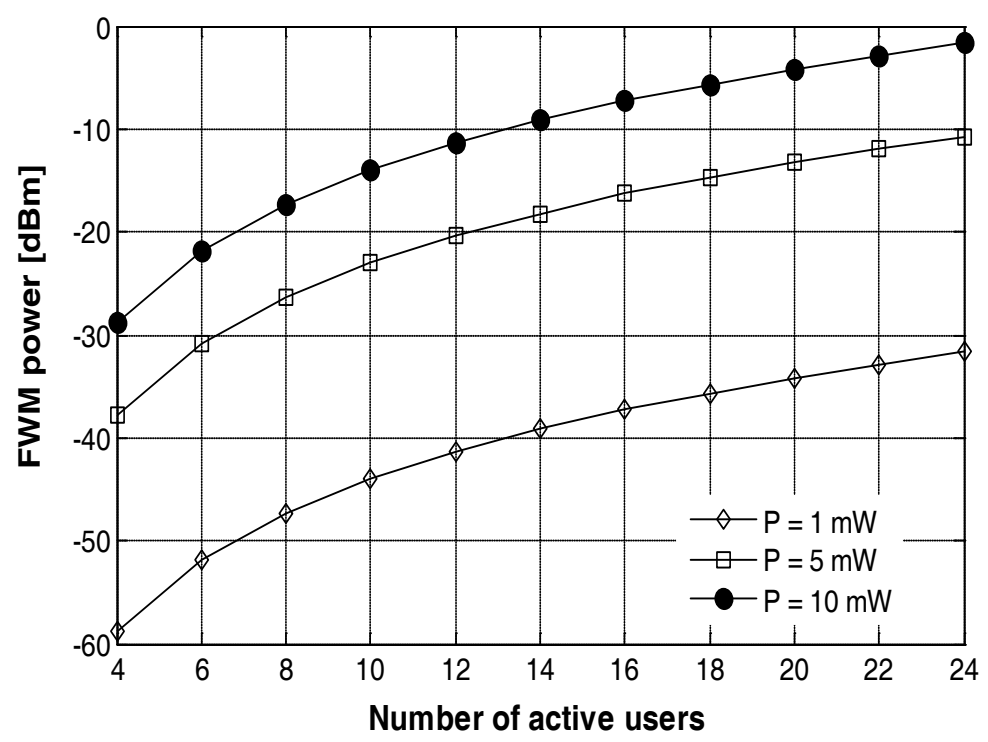

Fig. 3 FWM power versus the number of active users for different chip power when $w=7$ and $L=5 \mathrm{Km}$

The dependency of FWM-noise power on the fiber length for different types of optical fibers is illustrated in Fig.4. Three optical fibers are used in such analysis with different nonlinear characteristics; nonzero dispersion shifted fiber (NZ-DSF), standard single mode fiber (STD Corning SMF-28) and non-zero dispersion fiber (NZDF). 


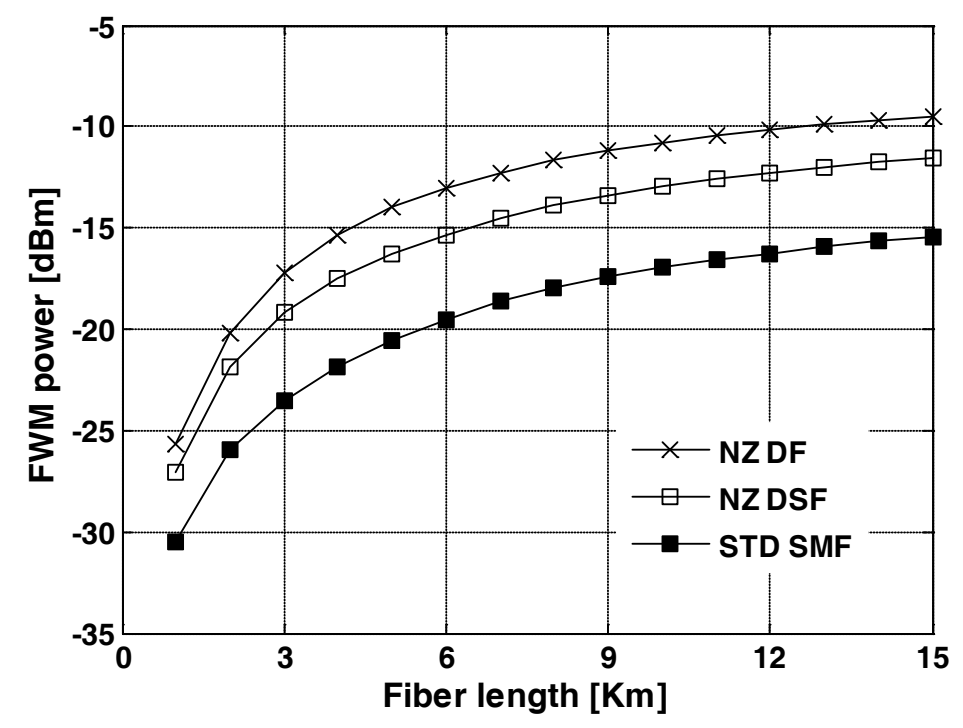

Fig. 4 FWM power versus fiber length for different fiber types when $w=7$ and $K=10$

Fig. 4 shows that the power of FWM increases with fiber length. As expected, STD SMF is less prone to FWM since its dispersion property reduces the phase matching between the mixed waves and hence decreasing the efficiency of FWM. On the other hand, DSF types are most affected by FWM because the values of fiber nonlinear parameter are high and consequently the efficiency of FWM generation is large. A trade-off analysis is required for the choice of the fiber type in terms of application length, group velocity and FWM effect. The effect of injected chip power for different wavelength spacing is illustrated in Fig. 5.

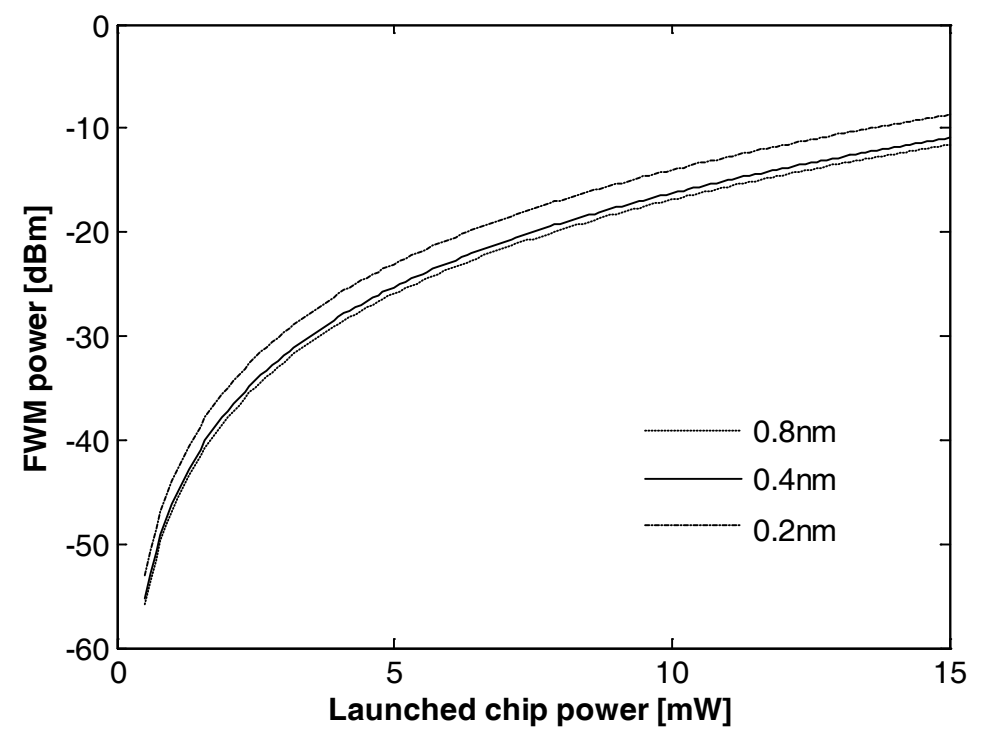

It is

Fig. 3 FWM power versus launched chip power for different wavelength spacing when $w=7, K=10$, and $L=5 \mathrm{Km}$ 
Military Technical College

Kobry Elkobbah,

Cairo, Egypt

April 19-21,2016

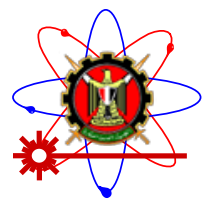

$8^{\text {th }}$ International Conference on

Mathematics and Engineering

Physics (ICMEP-8)

shown that when more chip power is launched through the fiber, the system gets affected by FWM noise power. Moreover, FWM efficiency is lower in case of wavelength spacing of $0.8 \mathrm{~nm}$ but such a high spacing requires large bandwidth which will has a negative impact on the system performance. The figure shows around 3dB difference in FWM power is observed between the wavelength spacing 0.2 and $0.8 \mathrm{~nm}$ at launched chip power of $5 \mathrm{~mW}$ and this difference becomes nearly constant for higher injected power. However, the presented analysis will provide useful material in system performance assessment under the presence of FWM.

\section{Conclusion}

A general formula for the generated FWM power in 2-D TW OCDMA systems is introduced. Analytical expressions of the number of FWM products on each desired wavelength and, consequently, the total number of FWM components utilizing symmetric codes with equally spaced wavelengths have been presented. The number of generated FWM components depends not only on the code weight but also on the number of users exhibiting an exponential growth in the FWM terms especially in middle wavelengths of the code weight. When $K=6$, more than 4 times rise in the FWM products when the code weight increases from 3 to 5 . In addition, the FWM-noise power behavior is very sensitive to 2-D TW OCDMA system parameters including injected power, number of active users, wavelength spacing, fiber type and length. Numerical results reveal that, as an example, increasing the launched chip power from 1 to $5 \mathrm{~mW}$ will result in an increase of FWM power by $20 \mathrm{~dB}$.

\section{References}

[1] L. Tančevski and I. Andonovic, "Wavelength-hopping time-spreading code division multiple access systems,” Electron. Lett., vol. 30, no. 17, 1994, pp. 1388-1390.

[2] M. Bharti, M. Kumar, and A. Sharma, "Design and analysis of OCDMA system using W/T codes at different bit rates," International Conference on Communication, Information and computation technology, ICCICT, 2015, pp. 1-5.

[3] I. Kaminow and T. Li, “Optical fiber telecommunications: Systems and impairments" Academic Press, 2002.

[4] N. Shibata, R. Braun and R. Waarts, "Phase-Mismatch Dependence of Efficiency of Wave Generation through Four-Wave Mixing in a Single-Mode Optical Fiber”, IEEE J. of Quantum Electronics, vol. 23, no. 7, 1987, pp. 1205-1210.

[5] M. Ajmani and P. Singh, "FWM in WDM System, Effects and Techniques to Minimize: A Review," International Conference on Advanced Computing and Communication Technologies, ACCT, 2015, pp. 385-389.

[6] N. Dang and A. Pham, "Performance analysis of incoherent multiwavelength OCDMA systems under the impact of four-wave mixing," OPTICS EXPRESS, vol. 18, no. 10, 2010, pp. 9922- 9933.

[7] H. Fenfei and S. Xiaohan, "Research on the FWM of optical CDMA Transmission System,” Microwave conference, 2008, pp. 599-602. 